\title{
A Generalized Mathematical Study of Two Phase Pulmonary Blood Flow in Lungs with Special Reference to Asthma
}

\author{
Manoj Kr. Srivastava*, V. Upadhyay*, A.K. Agrawal*, and P.N. Pandey** \\ *Department of Physical Sciences, M.G.C. Gramoday Vishwavidyalay, Chitrakoot, Satna, India. \\ ** Department of Mathematics, University of Allahabad, Allahabad, India
}

\begin{abstract}
In the present paper, we formulate the pulmonary blood flow in Lungs. Keeping in view the nature of pulmonary circulatory system in human body, the viscosity increases in the arterioles due to formation of roulex along axis by red blood cells, as we know the Lungs are remote from heart and proximate to the Asthma. P.N. Pandey and V. Upadhyay have considered the blood flow of two phased, one of which is that of red blood cells and other is Plasma. They have also applied the Herschel Bulkley non-Newtonian Model in bio-fluid mechanical set-up. We have collected a clinical data in case of Asthma for Hematocrit v/s Blood Pressure. The graphical presentation for particular parametric value is much closer to the clinical observation. The overall presentation is in tensorial form and solution technique adapted is analytical as well as numerical. The role of Hematocrit is explicit in the determination of blood pressure in case of pulmonary disease-Asthma.
\end{abstract}

\section{Introduction (Description of Bio-Physical Problem)}

Blood is a complex fluid consisting of particulate solids suspended in a non-Newtonian fluid. The particulate solids are red blood cells (RBCs), white blood cells (WBCs) and platelets. The fluid is plasma, which itself is a complex mixture of proteins and other intergradient in an aqueous base. $50 \%$ of the plasma and $45 \%$ of the blood cells and in $45 \%$ of the blood is RBCs and there is a few part of the other cells. Which are ignorable, so one phase of the blood is plasma and 2nd phase of the blood is RBCs.

Two phase pulmonary blood flow is a study of measuring the blood pressure if the hemoglobin is known. The percentage of volume covered by blood cells in the whole blood is called hematocrit. This work will focus on two phase pulmonary blood flow in Lungs with special reference to Asthma.

A lot of work is available, but P.N. Pandey and V. Upadhyay [5] discussed some phenomena in two phase blood flow gave an idea on the two phase pulmonary blood flow in Lungs with a pulmonary disease Asthma. The work of P.N. Pandey and V. Upadhyay is in whole circulatory system but our work will focus on pulmonary circulatory system, and pulmonary circulatory system which is a sub system of whole circulatory system. In this work, we apply the Herschel Bulkley non-Newtonian model. Here, pulmonary blood flow means blood flow in Lungs tissue. We present an improvement on the previous work in the field and this is discussed separately below. The ultimate use of this model is to predict normal reference levels of two phase blood flow in Lungs for individual patients undergoing to Asthma disease.

Recent research estimates that the number of people diagnosed with Asthma will rise from 23.7 million to 44.1 million between 2009 and 2034. The Centers for Disease Control and Prevention (CDCP) further predict that up to one-third of U.S. adults could have Asthma by 2050 if Americans continue to gain weight and avoid exercise. According to recent estimates, approximately 285 million people worldwide (6.6\%) in the 20-79 year age group have Asthma in 2010 and by 2030, 438 million people (7.8\%) of the adults population, is expected to have Asthma.

According to A.C. Guyton [11 ] the pulmonary circulation occurs due to blood pressure gradient, the high pressure of glomerular capillaries about $60 \mathrm{~mm} \mathrm{Hg}$ causes rapid fluid filtration, whereas much lower hydrostatic pressure in the particular capillaries about $13 \mathrm{~mm} \mathrm{Hg}$ permits rapid re-absorption. Pulmonary blood flow is not a linear function of the blood pressure gradient. The efferent Lungs are blood vessels that are part of the urinary tract of organisms. The efferent Lungs form from a convergence of the capillaries of the glomerular. They play an important role in maintaining the glomerular filtration rate despite fluctuations in blood pressure.

\section{Mathematical Modeling}

Let us the problem of blood flow in pulmonary circulatory system which is different from the problems in cylindrical tube and select generalized three dimensional orthogonal curvilinear coordinate system. Briefly described as $\mathrm{E}^{3}$ called as Euclidean space. According to Mishra [ 12], the biophysical laws thus expressed fully hold good in any co-ordinate system which is a compulsion for the truthfulness of the laws. According to I.W. Sherman and V. G. Sherman [3 ], blood is mixed fluid. Mainly there are two phases in blood. The first phase is plasma, while the other phase is that of blood cells are enclosed with a semi permeable membrane whose density is greater than that of plasma. These blood cells are uniformly distributed in plasma. Thus, blood can be considered 
as a homogeneous mixture of two phases .

\subsection{Equation of Continuity for two phase blood flow}

According to P. Singh and K. S. Upadhyay, the flow of blood is effected by the presence of blood cells. This effect is directly proportional to the volume occupied by blood cells. Let the volume portion covered by blood cells in unit volume be $\mathrm{X}$, this $\mathrm{X}$ is replaced by $\mathrm{H} / 100$, where $\mathrm{H}$ is the Hematocrit the volume percentage of blood cells. Then the volume portion covered by the plasma will be 1-X. If the mass ratio of blood cells to plasma is $r$ then clearly.

$$
r=\frac{X p c}{(1-X) P p}
$$

where $\mathrm{Pc}$ and $\mathrm{Pp}$ are densities of blood cells and blood plasma respectively. Usually this mass ratio is not a constant, even then this may be supposed to constant in present context.

The both phase of blood, i.e. blood cells and plasma move with the common velocity. Campbell and Pitcher have presented a model for this situation. According to this model, we consider the two phase of blood separately. Hence, the equation of continuity for two phase according to the principle of conservation of mass defined by J. N. Mishra and R.C. Gupta as follows:

$$
\frac{\partial(X p c)}{\partial t}+\left(X P c V^{i}\right), i=0
$$

and

$$
\left.\frac{\partial(1-X) P p}{\partial t}+(1-X) P p v^{i}\right), i=0
$$

where $\mathrm{v}$ is the common velocity of two phase blood cells and plasma. Again $\left(\mathrm{Xpcv}{ }^{\mathrm{i}}\right)$ is co-variant

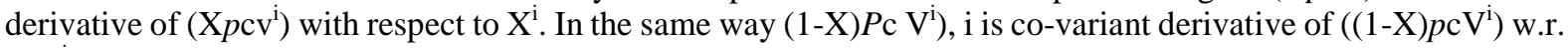
to $X^{\mathrm{i}}$

If we define the uniform density of the blood $P m$ as follow:

$$
\frac{1+r}{p m}=\frac{r}{p c}+\frac{1}{P p}
$$

Then, equations (2) and (3) can be combined together as follows:

$$
\frac{\partial p m}{\partial t}+\left(P m v^{i}\right), i \quad=0
$$

\subsection{Equation of Motion for two phase blood flow}

According to T.C. Ruche and H.D. [ 10 ], the hydro dynamical pressure p between the two phases of blood can be supposed to be uniform because the both phases i.e. blood cells and plasma are always in equilibrium state in blood. Taking viscosity coefficient of blood cells to be $n_{c}$ and applying the principle of conservation of momentum, we get the equation of motion for the phase of blood cells as follows:

$$
X p c \frac{\partial v i}{\partial t}+\left(X p_{c} V^{j}\right) V_{j}^{i}=-X p, j g^{i j}+X \eta \mathrm{c}\left(\mathrm{g}^{\mathrm{jk}} V_{k}^{i}\right), j
$$

Similarly, taking the viscosity coefficient of plasma to be..., the equation of motion for plasma will be as follows

$$
(1-X) P p \frac{\partial v^{i}}{\partial t}+\left\{(1-X) P p V^{i}\right\} V_{j}^{i}=-(1-X) p, j g^{j j}+(1-X) p, j g^{i j}+(1-X) \eta \mathrm{c}\left(\mathrm{g}^{\mathrm{jk}} V_{k}^{i}\right), j
$$

Now, adding equations (6) and (7) and using relation (4), the equation of motion for blood flow with the both phases will be as follows :

$$
P m \frac{\partial v^{i}}{\partial t}+\left(P m V^{j}\right) \nu_{j}^{i}=-p, j+\eta_{\mathrm{m}}\left(g^{j k} v_{k}^{i}\right), j
$$

where $\eta m=X \eta c+(1-X) \eta_{\mathrm{p}}$ is the viscosity coefficient of blood as a mixture of two phase. As the velocity of blood flow decreases, the viscosity of blood increases. The velocity of blood decreases successively 
because of the fact that Lungs, veinules and veins. These vessels are relatively a far enough from the heart. Hence the pumping of the heart on these vessels is relatively low [7]. Secondly, these vessels relatively narrow down more rapidly. In this situation, the blood cells line up on the axis to build up rouleaux. Hence a yield stress is produced. Though this yield stress is very small, even then the viscosity of blood is increased nearly ten times [31]. The Herschel Bulkley law holds good on the two phase blood flow through veins Lungs, veinules and whose constitutive equation is as follows :

$$
\begin{aligned}
& T^{\prime}=\eta_{m} e^{n}+T_{p}\left(T^{\prime} \geq T_{p}\right) \text { and } \\
& e=o\left(T^{\prime}<T_{p}\right) \text { where, }
\end{aligned}
$$

$T_{p}$ is the yield stress. When strain rate $e=o\left(T^{\prime} \geq T_{p}\right)$ a core region is formed which flows just like a plug. Let the radius of the plug be $r_{p}$. The stress acting on the surface of plug will be $T_{p}$. Equating the forces acting on the plug, we get [fig (1)]

$$
\begin{aligned}
\mathrm{P} \pi \pi_{\mathrm{p}}^{2}=\mathrm{T}_{\mathrm{p}} 2 \pi \mathrm{r}_{\mathrm{p}} & \\
& \\
& =>r p=2 \frac{T p}{P}
\end{aligned}
$$

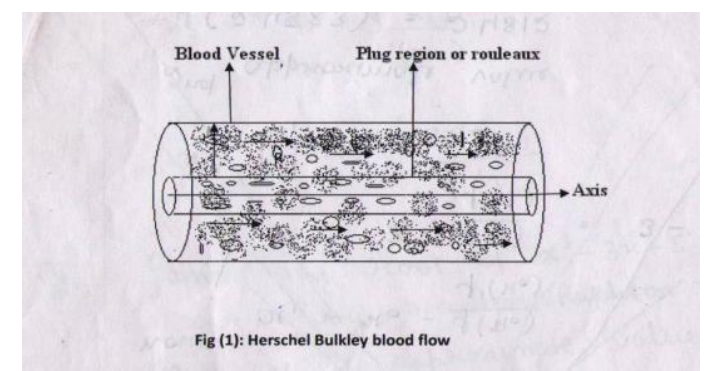

The Constitutive equation for test part of the blood vessel is

$\mathrm{T}^{\prime}=\eta \mathrm{me}^{\mathrm{n}}+\mathrm{T}_{\mathrm{p}} \quad$ Or $\mathrm{T}^{\prime}-\mathrm{T}_{\mathrm{p}}=\eta_{\mathrm{m}} \mathrm{e}^{\mathrm{n}}=\mathrm{T}_{\mathrm{e}}$

Where, $\mathrm{Te}=$ effective Stress

Whose generalized form will be as follows"

$$
T^{i j}=-P g^{i j}+T_{e}^{i j} \text { where }, T_{e}^{i j}=\eta_{m}\left(e^{i j}\right)^{n} \quad \text { While } e^{i j}=g^{j k} V_{k}^{i}
$$

Where the symbols have their usual meanings.

Now we describe the basic equation for Herschel Bulkley blood flow as follows :

$$
\text { Equation of Continuity - } 1 / \square \mathrm{g}(\square \text { gvi })_{, \mathrm{i}}=0 \text {. }
$$

Equation of Motion -

$$
p_{m} \frac{\partial v i}{\partial t}+P m V^{i} V_{, j}^{i}=-T_{e, j}^{i j}
$$

where all the symbols have their usual meanings.

\subsection{Solution and Discussion}

Since, the blood vessels are cylindrical; the above governing equations have to be transformed into cylindrical co-ordinates. As we know earlier:

$$
X^{1}=r, X^{2}=\theta, X^{3}=Z \text {, }
$$

Matrix of metric tensor in cylindrical co-ordinates is as follows :

$\left[g_{i j}\right]=\left[\begin{array}{ccc}1 & 0 & 0 \\ 0 & r^{2} & 0 \\ 0 & 0 & 1\end{array}\right]$

While matrix of conjugate metric tensor is as follows :

$$
\left[g^{i j}\right]=\left[\begin{array}{ccc}
1 & 0 & 0 \\
0 & \frac{1}{r^{2}} & 0 \\
0 & 0 & 1
\end{array}\right]
$$


Whereas the chritoffel's symbols of $2^{\text {nd }}$ kind are as follows :

$\left\{\begin{array}{cc}1 & \\ 2 & 2\end{array}\right\}=-r,\left\{\begin{array}{cc}1 \\ 2 & 2\end{array}\right\}=\left\{\begin{array}{cc}1 \\ 2 & 2\end{array}\right\}=\frac{1}{r}$

remaining others are zero.

Relation between contra variant and physical components of velocity of blood flow will be as follows :

$\sqrt{\mathrm{g}_{11 \mathrm{v}}{ }^{1}}=\mathrm{v}_{\mathrm{r}}=>\mathrm{v}_{\mathrm{r}}=\mathrm{v}^{1}$

$\sqrt{\mathrm{g}_{22 \mathrm{v}}{ }^{2}}=\mathrm{v}_{\theta}=>\mathrm{v}_{\theta}=r v^{2}$

$\sqrt{\mathrm{g}_{33 \mathrm{v}}{ }^{3}}=\mathrm{v}_{z}=>\mathrm{v}_{z}=v^{3}$

Again the physical components of $-\mathrm{p}, \mathrm{j}, \mathrm{ij}^{\mathrm{ij}}$ are $-\sqrt{g_{i j}} P, g^{i j}$

Equation (9) and (10) are transformed into cylindrical form so as to solve them as power law model to get

$\frac{d v}{d r}=\left(\frac{\mathrm{Pr}}{2 \eta_{m}}\right)^{\frac{1}{n}}$

Where, pressure gradient $\frac{d p}{d z}=P$

$\frac{d v}{d r}=\left(\frac{p\left(r-r_{p}\right)}{2 \eta_{m}}\right)^{\frac{1}{n}}$

$\frac{d v}{d r}=\left(\frac{\frac{1}{2} p r-\frac{1}{2} p r_{p}}{\eta_{m}}\right)^{\frac{1}{n}}$

From equation (9)

$\frac{d v}{d r}=\left(\frac{\frac{1}{2} p r-T_{p}}{\eta_{m}}\right)^{\frac{1}{n}}$

Substituting the value of $\mathrm{T}_{\mathrm{p}}$ from (7) into (11), we get

$$
\begin{aligned}
& \frac{d v}{d r}=\left(\frac{\frac{1}{2} p r-\frac{1}{2} \mathbf{P r}_{p}}{\eta m}\right)^{\frac{1}{n}} \\
& \frac{d v}{d r}=-(p / 2 \eta m)^{\frac{1}{n}}\left(r-r_{p}\right)^{\frac{1}{n}}
\end{aligned}
$$

Integrating above equation (12) under the no slip boundary condition: $\mathrm{v}=0$ at $\mathrm{r}=\mathrm{R}$ so as to get :

$$
V=\left(P / 2 \eta_{m}\right)^{\frac{1}{n}} \frac{n}{n+1}\left[\left(R-r_{p}\right)^{\frac{1}{n}+1}-\left(r-r_{p}\right)^{\frac{1}{n}+1}\right]
$$

This is the formula for velocity of blood flow in Lungs veinules and veins. Putting $r=r_{p}$ to get the velocity $V_{p}$ of plug flow as follows :

$$
V_{p}=\frac{n}{n+1}(P / 2 \eta m)^{\frac{1}{n}}\left(R-r_{p}\right)^{\frac{1}{n}+1}
$$

where the value of $r_{p}$ is taken from (7) 
A Generalized Mathematical Study Of Two Phase Pulmonary Blood Flow In Lungs With Special Reference

2.4. Result (Bio-physical interpretation)

Observations : Hematocrit vs. blood pressure from an authorized. Homeo Chikitsa Kendra, Allahpur by Dr.

Kamalesh Kumar Mishra.

Patient Name : Mr. Arun Singh.

Diagnosis : Asthma.

\begin{tabular}{|l|l|l|l|}
\hline Date & HB(Hemoglobin) & B.P. (blood pressure) & Hematocrit \\
\hline $10-01-11$ & 12.2 & $140.5 / 100$ & 36 \\
\hline $16-05-11$ & 11.8 & $130.5 / 90$ & 35.4 \\
\hline $20-05-11$ & 11.4 & $130.3 / 80$ & 33.6 \\
\hline $30-05-11$ & 12.3 & $120.2 / 80$ & 33 \\
\hline
\end{tabular}

The flow flux of two phased blood flow in Lungs, veinules and veins is

$$
\begin{aligned}
& \mathrm{Q}=\int_{0}^{\mathrm{r}_{\mathrm{p}}} 2 \pi \mathrm{rv} \mathrm{p}_{\mathrm{p}} \mathrm{dr}+\int_{\mathrm{r}_{\mathrm{p}}}^{\mathrm{R}} 2 \pi \pi \mathrm{rvd} \\
& =\int_{\mathrm{o}}^{\mathrm{r}_{\mathrm{p}}} 2 \pi \pi \frac{\mathrm{n}}{\mathrm{n}+1}\left(\frac{\mathrm{p}}{2 \eta \eta}\right)^{\frac{1}{\mathrm{n}}}\left(\mathrm{R}-\mathrm{r}_{\mathrm{p}}\right)^{\frac{1}{\mathrm{n}}+1} \mathrm{dr}+ \\
& \quad \int_{\mathrm{o}}^{\mathrm{r}_{\mathrm{p}}} 2 \pi \pi \frac{\mathrm{n}}{\mathrm{n}+1}(\mathrm{P} / 2 \mathrm{~nm})^{\frac{1}{\mathrm{n}}}\left[\left(\mathrm{R}-\mathrm{r}_{\mathrm{p}}\right)^{\frac{1}{\mathrm{n}}+1}-\left(\mathrm{r}-\mathrm{r}_{\mathrm{p}}\right)^{\frac{1}{n}+1}\right] \mathrm{dr}
\end{aligned}
$$

Using (12) and (14)

$$
\begin{aligned}
& \frac{2 \pi \mathrm{n}}{(\mathrm{n}+1)}\left(\frac{\mathrm{P}}{2 \eta \mathrm{m}}\right)^{\frac{1}{\mathrm{n}}}\left(\mathrm{R}-\mathrm{r}_{\mathrm{p}}\right)^{\frac{1}{\mathrm{n}}+1}\left[\frac{\mathrm{r}^{2}}{2}\right]_{0}^{\mathrm{r}_{\mathrm{p}}}+\frac{2 \pi \mathrm{n}}{(\mathrm{n}+1)}\left(\frac{\mathrm{P}}{2 \eta \mathrm{m}}\right)^{\frac{1}{\mathrm{n}}}\left[\frac{\mathrm{r}^{2}}{2}\left(\mathrm{R}-\mathrm{r}_{\mathrm{p}}\right)^{\frac{1}{\mathrm{n}}+1}-\frac{\mathrm{r}\left(\mathrm{r}-\mathrm{r}_{\mathrm{p}}\right)^{\frac{1}{\mathrm{n}}}}{\frac{1}{\mathrm{n}}+2}+\frac{\left(\mathrm{r}-\mathrm{r}_{\mathrm{p}}\right)^{\frac{1}{\mathrm{n}}+3}}{\left.\left(\frac{1}{\mathrm{n}}+2\right)\left(\frac{1}{\mathrm{n}}+3\right)\right]_{\mathrm{r}_{\mathrm{p}}}^{\mathrm{R}}}\right. \\
& \frac{\pi \mathrm{n}}{(\mathrm{n}+1)}\left(\frac{\mathrm{P}}{2 \eta \mathrm{m}}\right)^{\frac{1}{\mathrm{n}}} R^{\frac{1}{n}+3}\left[\frac{r^{\frac{2}{p}}}{R^{2}}\left(1-\frac{r^{\frac{2}{p}}}{R}\right)^{\frac{1}{n}+1}+\left(1+\frac{r_{p}}{R}\right)\left(1-\frac{r_{p}}{R}\right)^{\frac{1}{n}+2}-\frac{2\left(1-\frac{r_{p}}{R}\right)^{\frac{1}{n}+2}}{\left(\frac{1}{n}+2\right)}+\frac{2\left(1-\frac{r_{p}}{R}\right)^{\frac{1}{n}+3}}{\left(\frac{1}{n}+2\right)\left(\frac{1}{n}+3\right)}\right] \\
& \mathrm{Q}=425 \mathrm{ml} . / \min
\end{aligned}
$$

$\mathrm{R}=1, \quad \mathrm{r}_{\mathrm{p}}=\frac{1}{3}$

According to Gustafson, Daniel R. (1980)

$$
\eta_{p}=0.0013 \quad \text { (Pascal - sec.) }
$$

According to Glenn Elert (2010)

$$
\eta_{m}=0.027 \quad \text { (pascal- sec.) }
$$

$\mathrm{H}=24$

$\eta_{\mathrm{m}}=\eta_{\mathrm{c}} \mathrm{X}+\eta_{\mathrm{p}}(1-\mathrm{X})$ where, $X=\frac{\mathrm{H}}{100}=\frac{24}{100}=.24$

$1827.23=(1728.40)^{\frac{1}{n}}\left[\frac{26 n^{3}+33 n^{2}+9 n}{6 n^{3}+11 n^{2}+6 n+1}\right]$

Solved by Numerical method,

We get, $n=1.15$ 
From equation (5.1)

$$
\mathrm{P}=(.003213 \mathrm{H}+.0039)(1827.23)^{1.15} \times\left(\frac{26 n^{3}+33 n^{2}+9 n}{6 n^{3}+11 n^{2}+6 n+1}\right)^{n}
$$
At $\mathrm{H}=36, \quad \mathrm{P}=193.31$
At $\mathrm{H}=35.4, \mathrm{P}=190.1939$
At $\mathrm{H}=33.6, \mathrm{P}=180.8437$
At $\mathrm{H}=33, \quad \mathrm{P}=177.72$

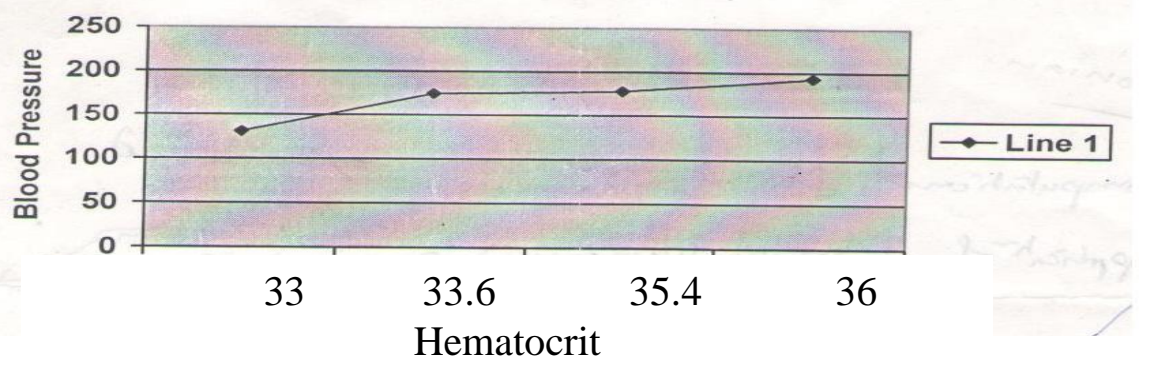

\section{Conclusion :}

A simple survey of the graph between blood pressure and hematocrit in Asthma patient shows that when Hematocrit is increased the Blood pressure also increased. Hence Hematocrit is proportional to blood pressure.

IV.

Acknowledgement :

I owe my sincere thanks to Dr. Kamalesh Kr. Mishra, Homeo Chikitsa Kendra Allahpur and thanks are due to Dr. Subodh Jain, toshi pathology centre Georgetown Allahabad.

Remark : If this would have been possible to get blood Pressure on the particular tissue (Lungs) then the relation between blood pressure and hemoglobin has been measured more accurately.

\section{References:}

[1]. Upadhyay V.; some phenomena in two phase blood flow; 2000

[2]. Debnath L. On a micro - continuum model of pulsatile blood flow, Act Mechanica 24 : 165 - 177, (1976),

[3]. Jones D.S. and Sleeman B.D. Differential Equation and Mathematical Biology6 G.A. and V., (1976),

[4]. Upadhyay V. and Pandey P.N. Newtonian Model of two phase blood flow in aorta and arteries proximate to the heart, Proc., of third Con. of Int Acad. Phy. Sci., (1999)

[5]. Upadhyay V. and Pandey P.N. A Power Law model of two phase blood flow in arteries remote form the heart, Proc. of thilrd con. of Int. Acad. Phy. Sci. (1999),

[6]. Singh P. and Upadhyay K.S. A new approach for the shock propagation in two - Phase system, Nat. Acad. Sci. Letters No. 2., (1985),

[7]. LKandau L.D. \& Liufchitz E.M. Fluid Mechanics, Pergamon Press., (1959),

[8]. Debnath L. On transient flows in Non-Newtonian liquids, Tensor N.S. : Vol. 27, (1973)

[9]. Kanpur J.N. Mathematical models in Biology and Medicine EWP press., (1985),

[10]. Ruch, T. C and H. D ;patton (ends) ; physiology and bio-physics-vols(ii) and (iii) W.B. S; 1973

[11]. A.C .Guyton and john E .hall, Medical physiology, $10^{\text {th }}$ edition ;saunders .

[12]. Mishra R .S .Tensors and Riemannion Geometry ,pothishala pvt, Ltd .Alld 1990 . 